Journal of Computer Science 7 (8): 1187-1193, 2011

ISSN 1549-3636

(C) 2011 Science Publications

\title{
Fault Tolerant Control Using Proportional-Integral-Derivative Controller Tuned by Genetic Algorithm
}

\author{
${ }^{1}$ S. Kanthalakshmi and ${ }^{2}$ V. Manikandan \\ ${ }^{1}$ Department of I and CE, PSG College of Technology, Coimbatore, India \\ ${ }^{2}$ Department of EEE, Coimbatore Institute of Technology, Coimbatore, India
}

\begin{abstract}
Problem statement: The growing demand for reliability, maintainability and survivability in industrial processes has drawn significant research in fault detection and fault tolerant control domain. A fault is usually defined as an unexpected change in a system, such as component malfunction and variations in operating condition, which tends to degrade the overall system performance. The purpose of fault detection is to detect these malfunctions to take proper action in order to prevent faults from developing into a total system failure. Approach: In this study an effective integrated fault detection and fault tolerant control scheme was developed for a class of LTI system. The scheme was based on a Kalman filter for simultaneous state and fault parameter estimation, statistical decisions for fault detection and activation of controller reconfiguration. Proportional-Integral-Derivative (PID) control schemes continue to provide the simplest and yet effective solutions to most of the control engineering applications today. Determination or tuning of the PID parameters continues to be important as these parameters have a great influence on the stability and performance of the control system. In this study GA was proposed to tune the PID controller. Results: The results reflect that proposed scheme improves the performance of the process in terms of time domain specifications, robustness to parametric changes and optimum stability. Also, A comparison with the conventional Ziegler-Nichols method proves the superiority of GA based system. Conclusion: This study demonstrates the effectiveness of genetic algorithm in tuning of a PID controller with optimum parameters. It is, moreover, proved to be robust to the variations in plant dynamic characteristics and disturbances assuring a parameter-insensitive operation of the process.
\end{abstract}

Key words: Fault tolerant control, PID tuning, based system, unexpected change, Kalman filter, genetic algorithms, controller reconfiguration, usually derive, system dynamics, control systems

\section{INTRODUCTION}

Modern technological systems rely on sophisticated control systems to meet improved performance and safety requirements. A conventional feedback control design for a complex system may result in an unsatisfactory performance, or even instability, in the event of fault occurrence. A fault is usually defined as an unexpected change in a system, performance malfunctions in actuators, sensors or other system components, which tends to degrade the overall system. In any process, the faults can be classified in two sets: Unrecoverable and recoverable. The unrecoverable ones represent all faults that cannot be compensated or accommodated while the system is running. On the other hand, the recoverable faults comprise any fault whose outcome can still be safely compensated by the control algorithm with a possible deterioration of performance, but still allowing the necessary conditions to maintain closed- loop stability. From a control point of view, the focus is on the recoverable faults, where a degree of robustness or reconfigurability in the control scheme is desirable to accommodate these faults and still preserve closed-loop performance. These types of control systems are often known as fault-tolerant control systems (FTCS), which possesses the ability to accommodate for system failures automatically and to maintain overall system stability and acceptable performance in the event of component failures. (Campos-Delgado et al., 2008; Niemann, 2004).

In general, fault tolerant control methods are classified into two types, i.e., Passive Fault Tolerant Control Scheme (PFTCS) and Active Fault Tolerant Control Scheme (AFTCS) (Mahmoud et al., 2003); Zhang, 2008). In PFTCS, controllers are fixed and are designed to be robust against a class of presumed faults. This approach needs neither FDD schemes nor controller reconfiguration, but it has limited faulttolerant capabilities. The main drawback of this scheme is the conservativeness that can be and CE, PSG College of Technology, Coimbatore, India 
incorporated; however, no extra complexity in the control implementation is carried out. The AFTCS react to the system component failures actively by reconfiguring control actions so that the stability and acceptable performance of the entire system can be maintained. The active FTC requires more computational power during implementations, but it can provide less conservative results and better closed-loop performance after faults. Typically, a AFTCS consists of three parts: A reconfigurable controller, a FDD scheme and a control law reconfiguration mechanism. (Zhang, 2002)

Many methods have been proposed for Fault Detection and Diagnosis (FDD) in dynamic systems. Fault detection based on modeling and estimation is one of the methods. The Kalman filter, one such model based approach, is a set of mathematical equations that provides an efficient computational (recursive) means to estimate the state of a process, in a way that minimizes the mean of the squared error (Yang, 1999). However, the Kalman filter is useful not only for state estimation but also for state prediction. It has been widely used in different fields during the past decades, such as on-line failure detection, real time prediction of vehicle motion and prediction for maneuvering target trajectories. The Kalman filter is a linear, discrete-time and finitedimensional system. Its appearance is a copy of the system that is estimated. Inputs of the filter include the control signal and the difference value between measured and estimated state variables. By minimizing mean-square estimation errors, the optimal estimate can be derived. As a result, the output of the filter becomes optimal estimates of the next step time-state variables.

The PID controller is the most common form of feedback in use today. According to an estimate nearly $90 \%$ of the controllers used in industries are PID controllers. The family of PID controllers is rightly known as the building blocks of control theory owing to their simplicity and ease of implementation (Giriraj et al., 2008). Designing and tuning a PID controller appears to be conceptually simple, but can be hard in practice, if multiple objectives are to be achieved. A conventional PID controller with fixed parameters may usually derive poor control performance when it comes to system complexities (Salami, 1995; Asriel, 1996). Since the gain and the time constants of the system change with the operating conditions the conventional PID controllers result in sub-optimal corrective actions and, hence, require frequent tuning adjustments. This stimulates the development of tools that can assist engineers to achieve the best overall PID control for the entire operating envelope of a given process.
Genetic Algorithms (GA) belonging to the families of evolutionary computational algorithms have been widely used in many control engineering applications. They are powerful optimization algorithms that work on a set of potential solutions, which is called population. GA finds the optimal solution through cooperation and competition among the potential solutions. These algorithms are highly relevant for industrial applications, because they are capable of handling problems with nonlinear constraints, multiple objectives and dynamic components-properties that frequently appear in realworld problems (Dionisio, 2005; Mechatronics, Monterey, California, USA, 801-806. 14. Voda and Landau, 1995). The PID controller is designed based on traditional tuning techniques and an introduction of GA is discussed in detail along with the implementation of the proposed GA algorithm. The simulation results are presented and justification given of the alternate PID controller based on time domain analysis and robustness.

\section{MATERIALS AND METHODS}

FDD scheme-kalman filter: The Kalman filter addresses the general problem of trying to estimate the state of a discrete-time controlled process that is governed by the linear stochastic difference equation:

$\mathrm{x}_{\mathrm{k}}=\mathrm{Ax}_{\mathrm{k}-1}+\mathrm{Bu}_{\mathrm{k}-1}+\mathrm{w}_{\mathrm{k}-1}$

With a measurement $\mathrm{z} \in \mathrm{R}^{\mathrm{m}}$ that is:

$\mathrm{z}_{\mathrm{k}}=\mathrm{Hx}_{\mathrm{k}}+\mathrm{v}_{\mathrm{k}}$

The random variables $\mathrm{w}_{\mathrm{k}}$ and $\mathrm{v}_{\mathrm{k}}$ represent the process and measurement noise (respectively). They are assumed to be independent (of each other), white and with normal probability distributions as in equations 3-4:

$\mathrm{P}(\mathrm{w}) \sim \mathrm{N}(0, \mathrm{Q})$

$\mathrm{P}(\mathrm{v}) \sim \mathrm{N}(0, \mathrm{R})$

In practice, the process noise covariance $\mathrm{Q}$ and measurement noise covariance $\mathrm{R}$ matrices might change with each time step or measurement, however here we assume they are constant. The nxn matrix A in the difference equation (1) relates the state at the previous time step K-1 to the state at the current step $\mathrm{K}$, in the absence of either a driving function or process noise. The nxl matrix $\mathrm{B}$ relates the optional 
control input $\mathrm{u} \in \mathrm{R}^{1}$ to the state $\mathrm{x}$. The mxn matrix $\mathrm{H}$ in the measurement Eq. (2) relates the state to the measurement $\mathrm{z}_{\mathrm{k}}$.

Let $\hat{x} \bar{k} \in R^{n}$ be the priori state estimate at step $k$ given the knowledge of process prior to step $\mathrm{k}$.

$\hat{\mathrm{x}} \mathrm{k} \in \mathrm{R}^{\mathrm{n}}$ be posterior state estimate at step $\mathrm{k}$ given measurement $\mathrm{Z}_{\mathrm{k}}$.

Then the priori and posterior estimate errors can be defined as in Eq. (5):

$\mathrm{e}_{\overline{\mathrm{k}}}=\mathrm{x}_{\mathrm{k}}-\hat{\mathrm{x}}_{\overline{\mathrm{k}}}$

$e_{k}=x_{k}-\hat{x}_{k}$

The a priori estimate error covariance is as represented in Eq. (6):

$\mathrm{P}_{\overline{\mathrm{k}}}=\mathrm{E}\left[\mathrm{e}_{\overline{\mathrm{k}}} \mathrm{e}_{\overline{\mathrm{k}}}^{\mathrm{T}}\right]$

And the a posterior estimate error covariance is as represented in Eq. (7):

$\mathrm{P}_{\mathrm{k}}=\mathrm{E}\left[\mathrm{e}_{\mathrm{k}} \mathrm{e}_{\mathrm{k}}^{\mathrm{T}}\right]$ (8):

A posterior state estimate is computed as in Eq.

$$
\hat{\mathrm{x}}_{\mathrm{k}}=\hat{\mathrm{x}}_{\overline{\mathrm{k}}}+\mathrm{K}\left(\mathrm{z}_{\mathrm{k}}-\mathrm{H} \hat{\mathrm{x}}_{\overline{\mathrm{k}}}\right)
$$

The difference $\left(Z_{k}-H_{\hat{x} \bar{k}}\right)$ is called the measurement innovation or the residual. The nxm matrix $\mathrm{K}$ in (8) is chosen to be the gain or blending factor that minimizes the a posterior error covariance and is given in (9):

$$
\mathrm{K}_{\mathrm{k}}=\mathrm{P}_{\mathrm{k}}-\mathrm{HT} /\left(\mathrm{HP}_{\mathrm{k}}-\mathrm{H}^{\mathrm{T}}+\mathrm{R}\right)
$$

The Kalman filter estimates a process by using a form of feedback control: the filter estimates the process state at some time and then obtains feedback in the form of (noisy) measurements. As such, the equations for the Kalman filter fall into two groups: time update equations and measurement update equations. The time update equations (predictor equations) are responsible for projecting forward (in time) the current state and error covariance estimates to obtain the a priori estimates for the next time step. The measurement update equations (corrector equations) are responsible for the feedback-i.e. for incorporating a new measurement into the a priori estimate to obtain an improved a posteriori estimate. The complete Kalman filter cycle is shown in Fig. 1.
PID controller: The PID controller is a well known controller widely used to improve the dynamic response as well as to reduce or eliminate the steady state error. The derivative controller adds a finite zero to the open loop transfer function and improves the transient response. The integral controller adds a pole at origin, thus increasing the system type by one and reducing the steady state error due to step function to zero. The PID controller consist of three type of control: proportional, integral and derivative which are combined together to form the transfer function as in Eq. (10):

$\mathrm{G}_{\mathrm{PID}}=\mathrm{K}_{\mathrm{d}} \mathrm{s}^{2}+\mathrm{K}_{\mathrm{p}} \mathrm{s}+\mathrm{K}_{\mathrm{i}} / \mathrm{s}$

A conventional PID controller with fixed parameters may usually derive poor control performance, since gain and time constants of the system change with the operating conditions and result in sub-optimal corrective actions and, hence require frequent tuning adjustments.

Zeigler-Nichols (ZN) tuning is straightforward and widely accepted method for tuning PID controller. Once the controller is tuned by ZieglerNichols method, generally, a good but not an optimum system response will be reached. So, it is desirable to increase the capabilities of PID controllers by any other effective tuning method. Many random search methods, such as Genetic Algorithm (GA) have recently received much interest for achieving high efficiency and searching global optimal solution in problem space.

GA based PID tuning: The optimal value of the PID controller Parameters $K_{P}, K_{i}$ and $K_{d}$ is to be found using the GA. All possible sets of controller parameter values are represented as chromosomes, whose values are adjusted to minimize the objective function, which is the Integral Square Error (ISE) criterion. Each chromosome consists of three separate strings constituting a P, I and D term, as defined by the 3-row 'bounds' declaration when creating the population. When the chromosome enters the evaluation function, it is split up into three terms, corresponding to $\mathrm{P}, \mathrm{I}$ and $\mathrm{D}$ gains.

The PID is tuned when the sum of the square error between the reference input test signal and the simulated system response inside the GA for PID tuning is close to zero. i.e., Objective Function to be formed as $\mathrm{J}=\int\left(1-\mathrm{Y}_{\mathrm{GA}}\right)^{2}$ for step input, where $\mathrm{y}_{\mathrm{GA}}$ is the internal GA simulation response of the system.

Additional code is added to ensure that the genetic algorithm converges to a controller that produces a stable system. If the poles of the controlled system are found to be in the right half of the s-plane, the error is assigned an extremely large value to make sure that the particular chromosome is not reselected. 


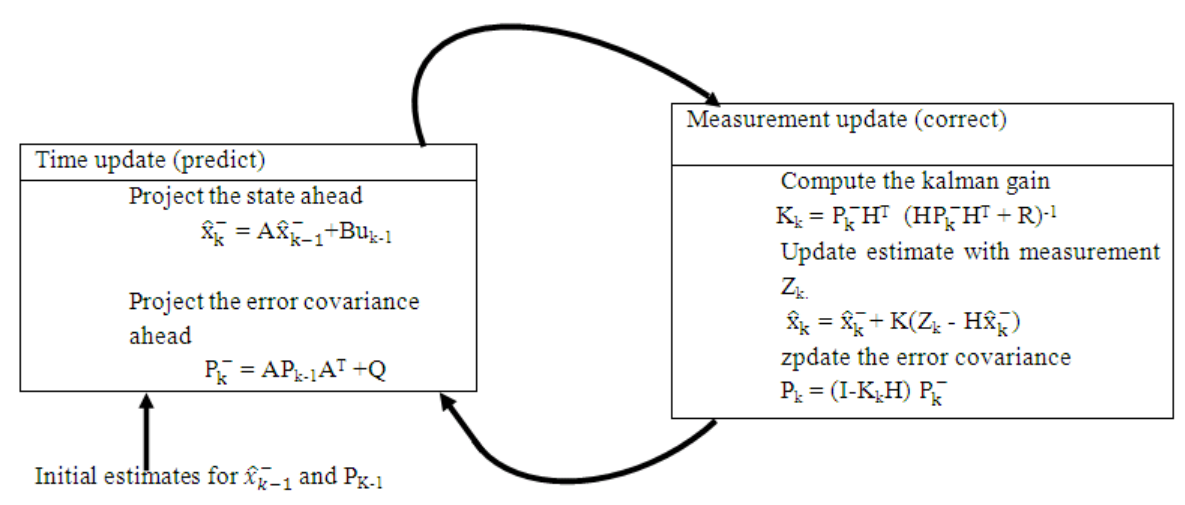

Fig. 1: Complete kalman filter cycle

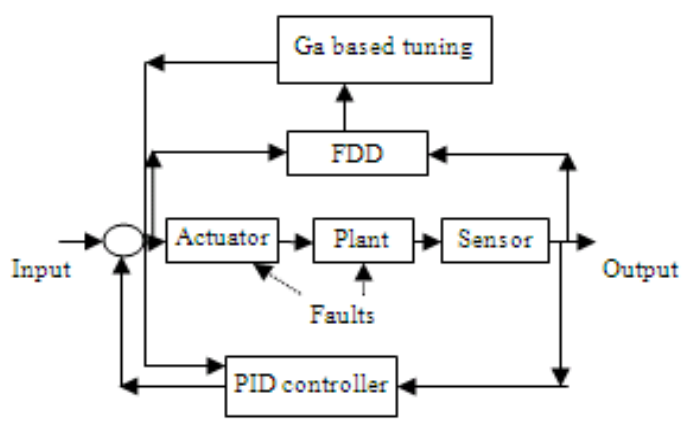

Fig. 2: Architecture of the proposed methodology

Proposed method: The architecture of the proposed method is shown in Fig. 2. The proposed Fault Tolerant Control (FTC) scheme is shown in Figure. The architecture of FTCS consist of the following major modules: Fault Detection and Diagnosis (FDD) using Kalman Filter, GA based Tuning and PID Controller. FDD module detect the fault either due to change in system dynamics or deterioration in the actuator performance. Based on the information from the FDD module, the GA tunes the PID Controller to maintain the satisfactory system performance.

Consider the Ball and hoop system represented in state space form as:

$$
\begin{aligned}
& {\left[\begin{array}{l}
\mathrm{x}_{1} \\
\mathrm{x}_{2}
\end{array}\right]=\left[\begin{array}{cc}
0 & \mathrm{I} \\
-\mathrm{M}^{-1} \mathrm{~K} & -\mathrm{M}^{-1} \mathrm{~L}
\end{array}\right]\left[\begin{array}{l}
\mathrm{X}_{1} \\
\mathrm{X}_{2}
\end{array}\right]} \\
& +\left[\begin{array}{c}
0 \\
\mathrm{M}^{-1} \mathrm{~B}
\end{array}\right] \mathrm{UY}=\mathrm{C}\left[\begin{array}{l}
\mathrm{X}_{1} \\
\mathrm{X}_{2}
\end{array}\right]+\mathrm{DU}
\end{aligned}
$$

Where:

$$
\begin{aligned}
& X=\left[\begin{array}{llll}
\theta & y & \theta & y^{\prime}
\end{array}\right]^{\mathrm{T}} \mathrm{M}=\left[\begin{array}{c}
\mathrm{I}_{\mathrm{a}}+\mathrm{m}(\mathrm{R}-\mathrm{r})^{2}-\mathrm{m}(\mathrm{R}-\mathrm{r})^{2} / \mathrm{R} \\
-\mathrm{m}(\mathrm{R}-\mathrm{r})^{2} / \mathrm{R} \mathrm{I}_{\mathrm{b}} / \mathrm{r}^{2}=(\mathrm{R}-\mathrm{r})^{2} / \mathrm{R}^{2}
\end{array}\right] \mathrm{L}= \\
& {\left[\begin{array}{cc}
\mathrm{b}_{\mathrm{m}} & 0 \\
0 & \mathrm{~b}_{\mathrm{b}} / \mathrm{r}^{2}
\end{array}\right] ; \mathrm{K}=\mathrm{mg}\left[\begin{array}{cc}
\mathrm{R}-\mathrm{r} & -(\mathrm{R}-\mathrm{r}) / \mathrm{R} \\
-(\mathrm{R}-\mathrm{r}) / \mathrm{R} & (\mathrm{R}-\mathrm{r}) / \mathrm{R}^{2}
\end{array}\right] ; \mathrm{B}=\left[\begin{array}{l}
1 \\
0
\end{array}\right]}
\end{aligned}
$$

The friction coefficient for the rotation of hoop $\mathrm{b}_{\mathrm{m}}=0.1$ :

- The friction coefficient for ball, $b_{b}=1.67 \mathrm{e}-6$

- Hoop radius $\mathrm{R}=0.085 \mathrm{~m}$

- Ball radius $r=0.0091 \mathrm{~m}$

- $\quad$ Mass of the ball, $\mathrm{m}=0.032 \mathrm{Kg}$

- Moment of inertia of the hoop, $\mathrm{I}_{\mathrm{a}}=1.6 \mathrm{e}-3$

- Moment of inertia of the ball, $\mathrm{I}_{\mathrm{b}}=1.059 \mathrm{e}-5$

- $\mathrm{G}=9.81 \mathrm{~m} \mathrm{~s}^{-1}$

With the above parameters substituted:

$$
\begin{aligned}
& \mathrm{A}=\left[\begin{array}{cccc}
0 & 0 & 1 & 0 \\
0 & 0 & 0 & 1 \\
-4.79 & 59.36 & -60.18 & -0.687 \\
7.045 & -88.88 & -3.407 & 10.5652
\end{array}\right] \\
& 34 \mathrm{~B}=\left[\begin{array}{lllll}
0 & 0 & 621.8 & 31.27
\end{array}\right]^{\mathrm{T}}
\end{aligned}
$$

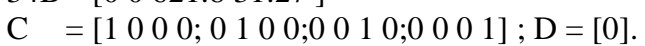

Any one of the state variables can be controlled, setting the output accordingly, while the most relevant variable that has crucial effect on the system performance is the ball position from the datum point. So, the output in this case is set as $\left[\begin{array}{llll}0 & 1 & 0 & 0\end{array}\right]$. The results obtained on applying the proposed methodology to the ball and hoop system is presented in the results.

\section{RESULTS AND DISCUSSION}

The system under ideal operating conditions is considered. The response of the system is estimated using the Kalman filter and compared with actual output, shown in Fig. 3. The Kalman filter is found to perform well as the estimate and actual values merge with each other. The Fig. 4 compares the system response (ball position) to step input with PID controller tuned by Zeigler-Nichols method and GA. 


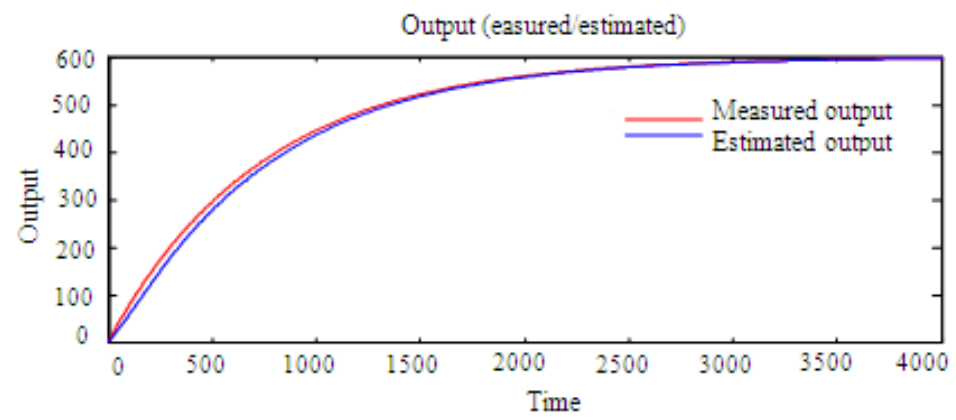

Fig. 3: System output under fault-free condition

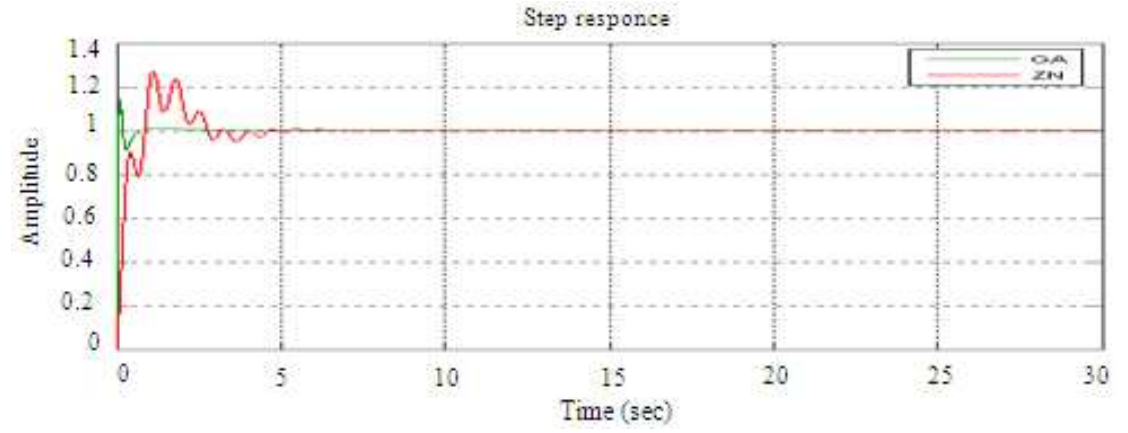

Fig. 4: GA and ZN responses under normal operation

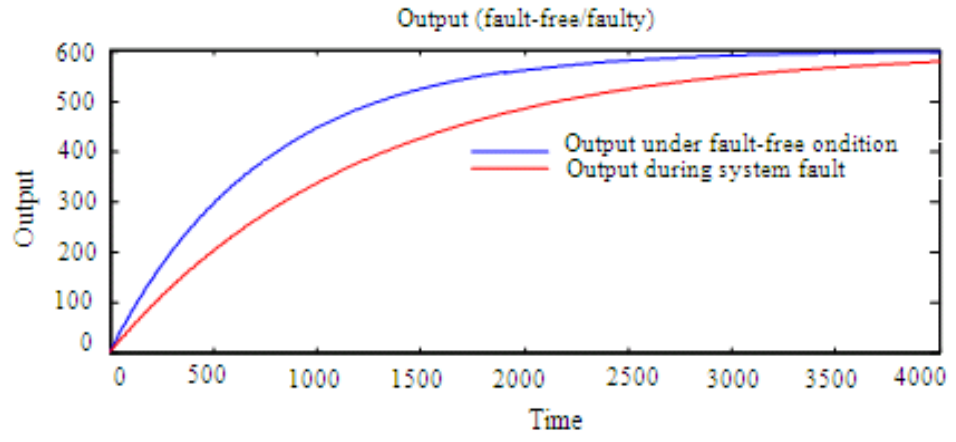

Fig. 5: System output under system fault condition

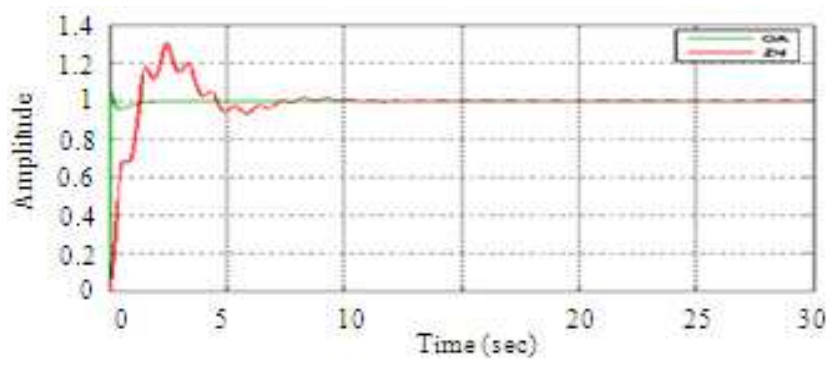

Fig. 6: GA and ZN responses after system fault condition 


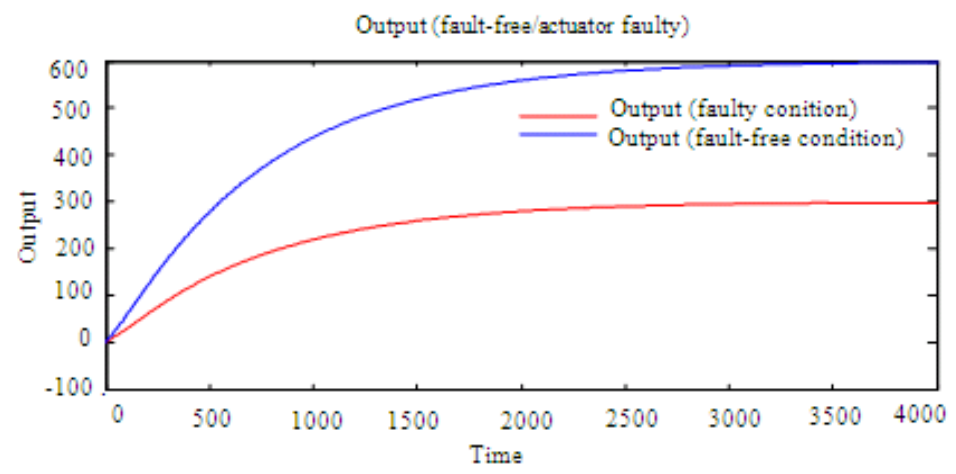

Fig. 7: System output under actuator fault condition

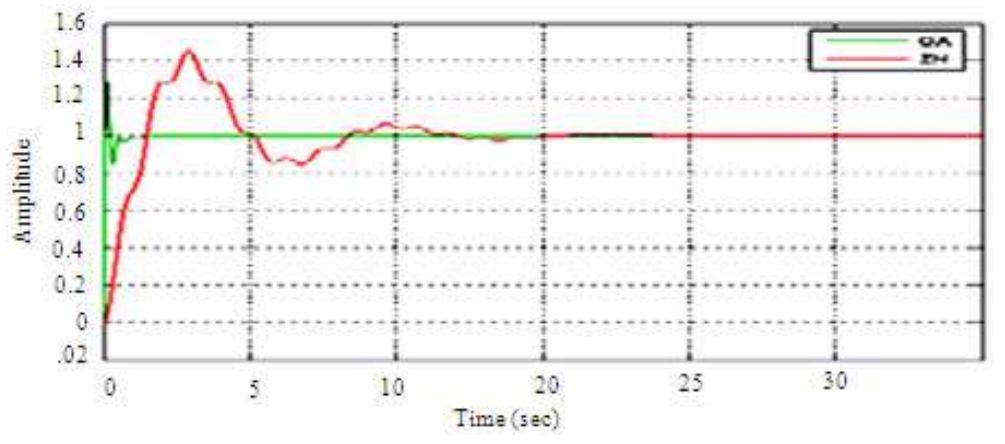

Fig. 8: GA and $\mathrm{ZN}$ responses under actuator fault condition

The dynamic characteristics are far better with minimal percentage overshoot $(15 \%)$ and settling time $(2.5 \mathrm{sec})$ when compared to Zeigler-Nichols method where the percentage overshoot is $27 \%$ and settling time is $10 \mathrm{sec}$.

As the system dynamics are changed, by altering the values of frictional coefficient of ball and hoop, the system response obtained using Kalman filter in comparison with actual fault-free system is shown in Fig. 5. The Fig. 6 compares the system response (ball position) to step input with PID controller tuned by Zeigler-Nichols method and GA

Next, a disturbance is added to the plant input in the form of motor torque (actuator faulty case) and response obtained using the Kalman filter is shown in Fig. 7.

The step responses for actuator fault using GA and ZN based PID controller is shown in Fig. 8.

These results prove the robustness of the proposed GA based tuning against both the system dynamic change and actuator fault condition.

\section{CONCLUSION}

The effectiveness of Kalman filter in fault detection and genetic algorithm in tuning PID is demonstrated in this study. The proposed scheme was tested against parametric changes in the ball and hoop system and actuator failure. The results showed that the GA based tuning is capable of adapting PID controller to physical plant dynamic characteristic changes. It was proved to be robust to the disturbances (actuator failure), assuring parameterinsensitive operation of the process. In comparison with conventional Ziegler-Nichols method, GA based method settled fast with minimum overshoot, even under disturbance and parametric variation. The only drawback was the longer execution time, which further increased with the population size and chromosome length. A medium population size of 100 and chromosome length of 80 resulted in an adequate convergence characteristics and accuracy of parameter consuming only reasonable execution time.

\section{REFERENCES}

Asriel, U.L. and K.S. Narendra, 1996. Control of nonlinear dynamical systems using neural networks. II. Observability, identification, and control. IEEE Trans. Neural Networks, 7: 30-42. DOI: $10.1109 / 72.478390$

Campos-Delgado, D.U., E. Palacios and D.R. Espinoza-Trejo, 2008. Fault Detection, Isolation, and Accommodation for LTI Systems Based on GIMC Structure. J. Contr. Sci. Eng., 2008: 1-15. DOI: $10.1155 / 2008 / 853275$ 
Giriraj Kumar, S.M., R. Jain, N. Anantharaman, V. Dharmalingam and K.M.M. Sheriffa Begum, 2008. Genetic algorithm based PID controller tuning for a model bioreactor, Indian chemical engineer. Ind. Inst. Chem. Eng., 50: 214-226. http://www.ice.org.in/vol50308/abs/vol50308/avi s/Giriraj.pdf

Niemann, H. and J. Stoustrup, 2004. Fault tolerant controllers for sampled-data systems. Proc. Am. Contr. Conf., 4: 3490-3495. http://ieeexplore.ieee.org/xpl/freeabs_all.jsp?arnu mber $=1384452$

Mahmoud, M., J. Jiang and Y. Zhang, 2003. Active Fault Tolerant Control Systems. Springer, Verlag.

Salami, M. and G. Cain, 1995. An adaptive PID controller based on genetic algorithm processor. Proceedings of the International Conference on Genetic Algorithms in Engineering Systems: Innovations and Applications, Sep. 12-14, IEEE Xplore Press, Sheffield, UK., pp: 88-93. DOI: 10.1049/cp:19951030
Voda, A.A. and I. D. Landau, 1995. A method for the auto-calibration of PID controllers. Automatica, 31: 41-53. DOI: 10.1016/0005-1098(94)00067-S

Yang, S.K. and T.S. Liu, 1999. State estimation for predictive maintenance using Kalman filter. Reliability Eng. Syst. Saf., 66: 29-39. DOI: 10.1016/S0951-8320(99)00015-0

Zhang, Y. and J. Jiang, 2008. Bibliographical review on reconfigurable fault-tolerant control systems. Ann. Rev. Contr., 32: 229-252. DOI: 10.1016/J.ARCONTROL.2008.03.008

Zhang, Y.M. and J. Jiang, 2002. Active fault-tolerant control system against partial actuator failures. IEEE Proc. Contr. Theory Appl., 149: 95-104. DOI: $10.1049 /$ IP-CTA:20020110 